\title{
Efficacy and safety of polydioxanone thread embedded at specific acupoints for non- specific chronic neck pain: a study protocol for a randomized, subject-assessor-blinded, sham-controlled pilot trial
}

\author{
Eunseok Kim ${ }^{1+}$, Hye Su Kim ${ }^{2+}$, So-Young Jung ${ }^{3}$, Chang Hyun $\mathrm{Han}^{3^{*}}$ and Young-ll Kim ${ }^{1 *}$ (1)
}

\begin{abstract}
Background: This study aims to evaluate the efficacy and safety of thread-embedding acupuncture (TEA) with polydioxanone thread embedded at various acupoints, compared with sham TEA, for the treatment of non-specific chronic neck pain.

Methods/design: This study will be an 8-week-long, two-armed, parallel, randomized, subject-assessor-blinded, shamcontrolled pilot trial. Fifty eligible patients will be randomly allocated into the real TEA group or the sham TEA group. The real TEA group will receive TEA treatment at 14 fixed acupoints in the neck region. The sham TEA group will receive the same treatment as the real TEA group, but with a sham device with the thread removed. Both groups will receive treatment once a week for a total of four sessions. The primary outcome will be the mean change in the visual analog scale (VAS) from baseline to week 6 ( 2 weeks post intervention). Clinical relevance (ratio of the number of patients with decreases on the VAS of $\geq 15 \mathrm{~mm}$ or with percentiles $\geq 30 \%$ and $\geq 50 \%$ relative to baseline to the total number of patients), Neck Disability Index, pressure pain threshold, the Hospital Anxiety and Depression Scale, EuroQol 5-Dimensions questionnaire, Patient Global Impression of Change, blinding test, and adverse events will be used to assess secondary outcomes.
\end{abstract}

Discussion: The results of this study will provide valuable data for a large-scale clinical trial to evaluate the clinical effects of polydioxanone TEA in the treatment of patients with non-specific chronic neck pain.

Trial registration: Clinical Research Information Service (CRIS), Republic of Korea, KCT0002452. Registered on 6 September 2017.

Keywords: Chronic neck pain, Polydioxanone, Thread-embedding acupuncture, Efficacy, Randomized shamcontrolled trial

\footnotetext{
*Correspondence: chhan@kiom.re.kr; omdkim01@dju.kr

${ }^{\dagger}$ Eunseok Kim and Hye Su Kim contributed equally to this work.

${ }^{3}$ Clinical Research Division, Korea Institute of Oriental Medicine, 1672,

Yuseongdae-ro, Yuseong-gu, Daejeon 34054, Republic of Korea

${ }^{1}$ Department of Acupuncture and Moxibustion Medicine, College of Korean

Medicine, Daejeon University, 62, Daehak-ro, Dong-gu, Daejeon 34520,

Republic of Korea

Full list of author information is available at the end of the article
}

(c) The Author(s). 2018 Open Access This article is distributed under the terms of the Creative Commons Attribution 4.0 International License (http://creativecommons.org/licenses/by/4.0/), which permits unrestricted use, distribution, and reproduction in any medium, provided you give appropriate credit to the original author(s) and the source, provide a link to the Creative Commons license, and indicate if changes were made. The Creative Commons Public Domain Dedication waiver (http://creativecommons.org/publicdomain/zero/1.0/) applies to the data made available in this article, unless otherwise stated. 


\section{Background}

Non-specific chronic neck pain (CNP) is a common health problem, affecting $30-50 \%$ of the general population, and $23 \%$ of those individuals with CNP develop a recurrent episode within a month after their recovery [1, 2]. Of adult Europeans, 19\% have chronic pain seriously affecting their daily activities and, of those, 20\% have neck pain [3]. In the United Kingdom, 29\% of adults experienced back or neck pain in the prior month, with half of those reporting chronic pain [4], and these observations are similar to those on a United States survey [5]. According to a survey conducted between 2007 and 2008 in Korea, the lifetime prevalence of neck pain is $20.8 \%$ [6]. CNP is also common in most occupational groups and is related to disability in both social and occupational groups because $11.0-14.1 \%$ of workers are limited in their activities each year [1, 7-9]. The economic burden associated with neck pain is increasing because many patients tend to continue to utilize health care resources for treatment for up to 10 years after the initial onset $[10,11]$.

Various non-surgical treatments, such as non-steroidal anti-inflammatory drugs (NSAIDs), muscle relaxants, analgesics, physiotherapy, interdisciplinary rehabilitation, education, and spinal injection, are available for the treatment of patients with CNP [12-14]. Although painrelieving medication is most frequently used to alleviate CNP, long-term NSAID use is limited due to the risk of side effects and patient intolerance $[15,16]$. On the other hand, acupuncture has been increasingly recognized as being clinically beneficial because it has been consistently reported to be effective and safe in reducing pain and improving quality of life for patients with neck pain [13, 17-19].

Thread-embedding acupuncture (TEA) is a special type of acupuncture designed to embed thread at targeted tissue by using a hollow needle. Absorbable suture materials, such as polydioxanone (PDO), catgut, PCL (polycaprolactone), etc., are used for the threads in the TEA devices [20]. PDO thread has been used in TEA devices in Korea while catgut thread is used in China. PDO thread is known to take approximately 180 days after implantation to be completely absorbed by the tissue [21]. TEA is considered to have longer therapeutic effects through continuous stimulation by the threads embedded at the acupoints.

Randomized clinical trials (RCTs) in China evaluating the effect of catgut thread embedded at certain acupoints for the treatment of patients with various diseases, polycystic ovary syndrome, obesity, and postmenopausal period, have been reported [22-24]. A previous study also demonstrated the safety and efficacy of catgut TEA for the treatment of allergic rhinitis by using a sham device [25]. In Korea, the embedding of PDO thread at certain acupoints is widely used in the clinical practice of Korean Medicine for the treatment of chronic musculoskeletal pain. Nevertheless, the current level of evidence supporting the efficacy of embedding with PDO thread for patients with chronic musculoskeletal pain is insufficient. Only case studies on the application of PDO TEA for the treatment of patients with chronic low back pain [26], shoulder pain [27], and osteoarthritis of the knee [28] have been published. In addition, no RCTs using PDO thread embedded as a sham-controlled intervention have been reported [29].

Therefore, we designed a pilot RCT to investigate the efficacy of PDO thread embedded at certain acupoints for the treatment of patients with CNP. In addition, adverse effects of PDO TEA will be evaluated for safety assessment.

\section{Method/design \\ Objective}

The primary objective of this pilot study is to assess the therapeutic efficacy of PDO thread embedded at certain acupoints for the treatment of patients with CNP, compared to sham TEA, and to obtain valuable data and insights for a confirmative, full-scale RCT.

\section{Design}

This study will be a single-center, equally randomized, two-armed, parallel, stratified (gender), subject-assessorblinded, sham-controlled pilot trial. This trial is being conducted at the Pain and Spine Center, Dunsan Korean Medicine Hospital of Daejeon University (DKMHDU), Korea.

A total of 50 participants diagnosed with CNP will be recruited from the Clinical Trial Center of DKMHDU in Daejeon through advertisements in local newspapers, on hospital websites and bulletin boards, in information leaflets, etc. The recruitment period will run from May 2017 to December 2017. Clinical research coordinators will interview all potential participants over the telephone and schedule screening visits. At the screening visits, all potential participants will be informed of the objectives and the overall procedure of the trial and will be asked to provide written informed consent. Study volunteers will go through a screening process, which will include radiography of the cervical spine and physical examinations, to verify the diagnosis of non-specific CNP.

At visit 1, an eligible participant will receive baseline assessments, including the use of the acupuncture expectancy scale $[30,31]$, and will then be randomly allocated into one of two groups: the real TEA group and the sham TEA group. The real TEA group will receive TEA treatment at local acupoints in the neck region. The sham TEA group will receive the same treatment as 
the real TEA group, but with a sham device with the thread removed. Both groups will receive treatment once a week for a total of four sessions. The real TEA and the sham TEA will be described to the participants as "classical TEA" and "non-classical TEA," respectively. Researchers will explain to the participants that the term "classical TEA" is "typically used in Korean Medicine clinics" while the term "non-classical TEA" is "rarely used in Korean Medicine clinics" [32, 33]. Outcome measures will be assessed before the treatment (week 1), 2 weeks after the first treatment (week 4), and 2 weeks (week 6) and 4 weeks (week 8) after the last treatment. A flowchart of the study is illustrated in Fig. 1, and the clinical trial schedule is presented in Fig. 2.

\section{Participants}

\section{Inclusion criteria}

A participant will be enrolled if they fulfill the following criteria: (1) age 19-65 years; (2) suffering from persistent or recurrent neck pain lasting longer than 3 months [34] (neck pain is defined as "pain, ache, or discomfort" in the area between the occiput and the third thoracic vertebra and between the medial borders of the scapulae [35]); (3) indicating pain of more than $40 \mathrm{~mm}$ on the visual analog scale (VAS) at the time of screening; (4) diagnosis of non-specific neck pain by a clinician based on history taking, physical examination, image examination, and medical examination; (5) able and willing to comply with the intervention and follow-up evaluation; and (6) able to provide written informed consent.

\section{Exclusion criteria}

Participants with any of the following conditions will be excluded: (1) radicular pain in the upper extremity with the distribution of a particular nerve root; (2) neurological abnormality: (a) weakness, paresthesia (sensory deficits), (b) positive sign on a special test: shoulder abduction relief sign test (Bakody sign), Spurling test, (c) hyporeflexia of the deep tendon reflex, (d) pathological reflex: Hoffman sign, ankle clonus, Babinski sign, (e) muscle atrophy; (3) major spinal pathology such as neoplasm, myelopathy, spondylitis, and congenital abnormality; (4) history of spinal surgery or scheduled for surgery during the study; (5) pain in another region that is more severe than the neck pain; (6) uncontrolled lumbar pain; (7) history of TEA treatment; (8) hypersensitive reaction to previous acupuncture treatment, metal allergy, keloid, severe atopy, and other skin hypersensitivities; (9) hemorrhagic disease and/or factors that can affect hemostasis, such as anti-coagulant or anti-platelet drug use; (10) pregnancy, lactation, or a plan to become pregnant during the study period; (11) uncontrolled diabetes, or major cardiovascular disease; (12) history of neurotic or major psychiatric disability or cognitive instability; (13) history of alcoholism or drug abuse; and (14) severe underlying disease requiring active therapy. Furthermore, participants considered to be inappropriate for the study by the researcher will be excluded.

\section{Randomization and blinding}

An independent, blinded statistician will generate the random number table by using the stratified randomization

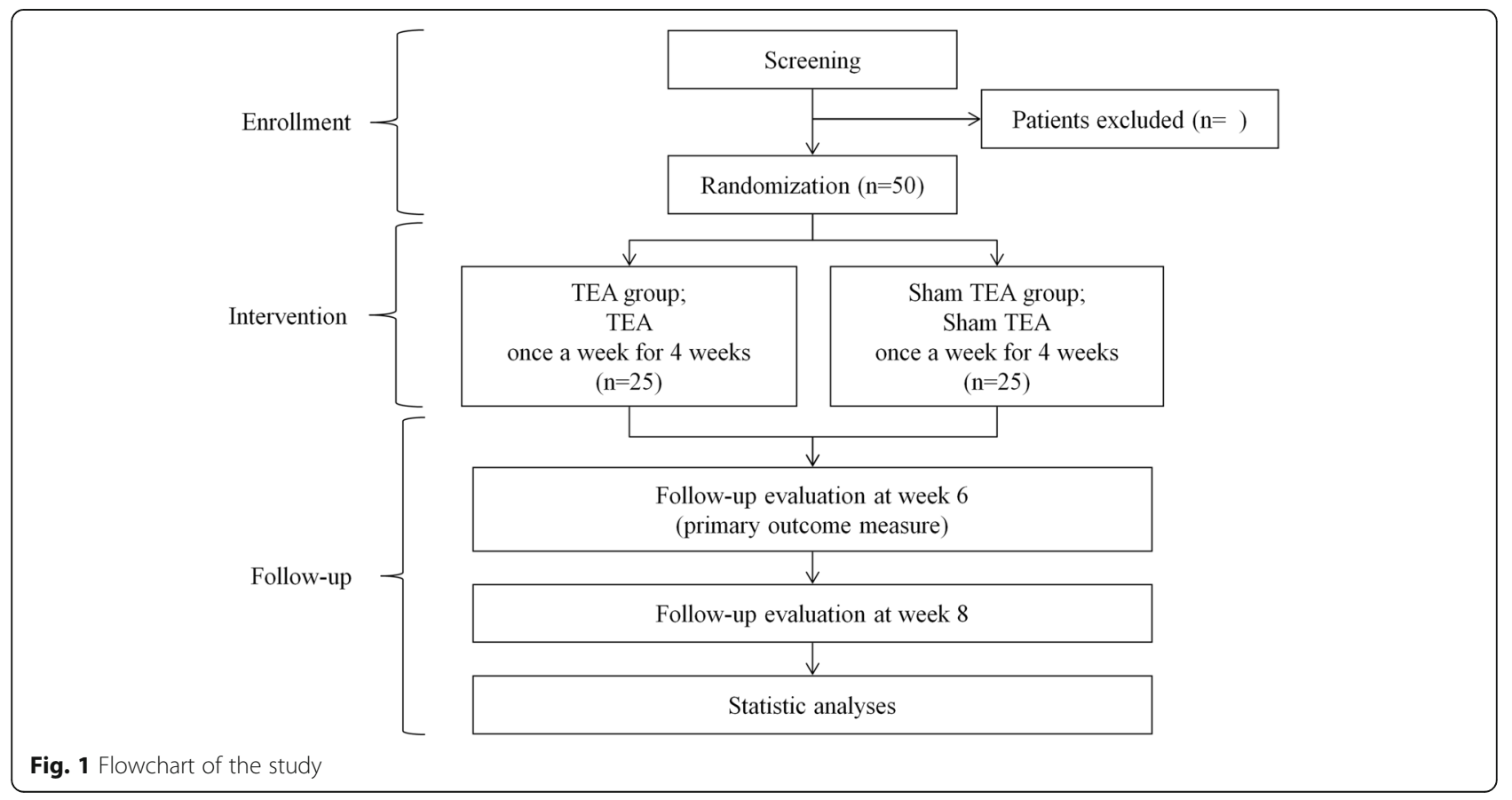




\begin{tabular}{|c|c|c|c|c|c|c|c|}
\hline \multirow[b]{3}{*}{ Timepoint } & \multicolumn{7}{|c|}{ Study period } \\
\hline & Enrollment & $\mathrm{V} 1$ & V2 & V3 & V4 & V5 & V6 \\
\hline & -2 & wk 1 & wk 2 & wk 3 & wk 4 & wk 6 & wk 8 \\
\hline \multicolumn{8}{|l|}{ ENROLMENT: } \\
\hline Informed consent & 0 & & & & & & \\
\hline $\begin{array}{l}\text { Demographic } \\
\text { characteristics } \\
\text { Medical history }\end{array}$ & 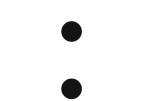 & & & & & & \\
\hline Physical examination & 0 & & & & & & \\
\hline $\begin{array}{l}\text { Radiography of cervical } \\
\text { spine } \\
\text { Blood test }\end{array}$ & 0 & & & & & - & \\
\hline Inclusion/Exclusion criteria & 0 & & & & & & \\
\hline $\begin{array}{l}\text { Treatment expectancy } \\
\text { questionnaire } \\
\text { Random allocation }\end{array}$ & 0 & 0 & & & & & \\
\hline \multicolumn{8}{|l|}{ INTERVENTIONS: } \\
\hline Real TEA treatment & & $\bigcirc$ & $\bigcirc$ & $\bigcirc$ & $\bigcirc$ & & \\
\hline Sham TEA treatment & & () & () & ( ) & (2) & & \\
\hline \multicolumn{8}{|l|}{ ASSESSMENTS: } \\
\hline VAS & 0 & ○ & & 0 & & 0 & 0 \\
\hline NDI & & 0 & & 0 & & ○ & 0 \\
\hline Pressure pain threshold & & ○ & & $\bullet$ & & 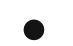 & 0 \\
\hline HADS & & $\bullet$ & & $\bullet$ & & $\bullet$ & $\bullet$ \\
\hline EQ-5D & & $\bullet$ & & $\bullet$ & & $\bullet$ & $\bullet$ \\
\hline PGIC & & & & & & 0 & 0 \\
\hline Blinding test & & - & & & $\bullet$ & & \\
\hline Safety assessment & & - & ○ & 0 & - & $\bullet$ & $\bullet$ \\
\hline
\end{tabular}

Fig. 2 Schedule for the treatment and outcome measurements

method of SAS ${ }^{\bullet}$ Analytics Pro (SAS Institute, Inc., Cary, NC, USA). The gender (male vs. female) will be applied as a condition value for the stratification. The random number table will be kept by the independent statistician and concealed with a password not to be exposed.

At visit 1, after baseline assessments, participants will be randomized into the experimental (real TEA treatment) group or the control (sham TEA treatment) group. Although the practitioner cannot be blinded, both participants and outcome assessors will be blinded to the allocation throughout the study period.

The study volunteers who have experience with TEA treatment will be excluded to prevent allocation guessing through discomforts, such as stiffness and irritation, post TEA treatment. Both the TEA treatment and the sham TEA treatment will be conducted by an individual practitioner, and identical treatment procedures in the same treatment room environments will be used. Because the sham TEA device appears identical to the real TEA device except for the thread hanging outside the needle, before starting the TEA treatment, the participants while in a sitting position will be instructed to turn their back on the practitioner to prevent them from being able to note the existence, or not, of the thread in the TEA needle.

\section{Interventions}

All subjects will receive a total of four sessions for the real TEA or the sham TEA (dry-needling) treatment, i.e., once a week for 4 weeks. To avoid any non-specific effect caused by interactions, the practitioner will not be allowed to give any positive or negative encouragement for future progress. For proper treatment, the practitioner will be allowed to conduct minimal examination of subjects, which include palpating the subject's neck to find the appropriate acupoints. All the treatment procedures and regimens will be described in detail in a prespecified protocol and standard operating procedures (SOPs) to ensure that the conditions experienced by both group, except for the TEA device, are identical. Both the real TEA treatment and the sham TEA treatment will be consistently performed by the same individual practitioner throughout the study period. The practitioner has completed 6 years of education for Traditional Korean Medicine and has at least 4 years of clinical experience with 
TEA. The practitioner was trained in the trial protocol and proficient in TEA treatment prior to the study. Both real and sham TEA treatment comply with the Standards for Reporting Interventions in Clinical Trials of Acupuncture (STRICTA) (see Additional file 1) [36]. Co-intervention will not be allowed except for rescue medicine (acetaminophen, maximum dose $3000 \mathrm{mg} /$ day). The exact dose of the rescue medicine will be recorded in the case report form at each visit.

\section{PDO TEA treatment}

The needle that will be used for TEA is made up of two parts: the external needle and the internal PDO thread. We will use disposable, sterile, PDO-threadembedding devices (OV World Co., Seoul, Republic of Korea) with 29-gauge (G) needles and USP size 6-0 PDO thread (Samyang Biopham Co., Seongnam-si, Republic of Korea). Two standard sizes of needle will be used: $29-\mathrm{G} \times 38-\mathrm{mm}$ needle with $54-\mathrm{mm}$ PDO thread $(27 \mathrm{~mm} \times 2$; folded in half) and $29-\mathrm{G} \times 25-\mathrm{mm}$ needle with $30-\mathrm{mm}$ PDO thread $(15 \mathrm{~mm} \times 2$; folded in half). One side of the PDO thread folded in half is inside the hollow needle while the other side of the thread is hanging outside the needle with fixation by the thread anchor (polystyrene). The threads will be embedded bilaterally at local acupoints in the neck region. The acupoints are selected on the basis of Meridian Theories, the text book [20], preceding studies on acupuncture for patients with CNP [37, 38], and the consensus of Korean acupuncture experts. The seven TEA points used in this study are GB20, TE16, LI17, GB21, and SI14 for regular acupoints and two paravertebral points, one each at the levels of the fifth and the seventh cervical vertebrae (C5 and C7), for extra acupoints (Table 1). The paravertebral points are located at 0.5 body-cun lateral to the spinous process.
The 29-G $\times 38-\mathrm{mm}$ needle will be applied at LI17, GB21 and paravertebral points at C7, and the $29-\mathrm{G} \times$ 25-mm needle will be used for GB20, TE16, SI14, and paravertebral points at C5. The TEA needles will be removed immediately after needle insertion, and manual manipulation for Deqi will not be allowed. The acupoints will be sterilized with a disposable $70 \%$ isopropyl alcohol swab prior to needle insertion. All procedures will be performed according to the Clean Needle Technique [39].

\section{Sham TEA treatment}

The sham TEA device will be a dry needle with the PDO thread removed. The sham TEA device will be made by the same manufacturer and will be, for all practical purposes, identical to the real TEA device except for the absences of the thread hanging outside the needle and the thread anchor (Fig. 3). For the sham TEA group, the entire TEA treatment procedure, such as number of acupoints, selection of needle size for each acupoint and needle-insertion method, will be the same as for the real TEA group. In other words, subjects in the control (sham TEA) group will receive only the stimulus of dry-needling, but not that of the embedded PDO thread.

\section{Outcome measurement}

Outcome measurements will be performed before the treatment (baseline; week 1), 2 weeks after the first treatment (week 3), and 2 weeks (week 6) and 4 weeks (week 8) after the last treatment.

\section{Primary outcome}

The primary outcome will be the mean change on the 100-mm VAS from the baseline (week 1) to the primary endpoint (week 6), which will be 2 weeks after the

Table 1 Locations of needling details for the acupoints used in the treatment protocol

\begin{tabular}{|c|c|c|}
\hline Acupoints & Location & Needling details \\
\hline GB20 (Fengchi) & $\begin{array}{l}\text { In the posterior region of the neck, inferior to the occipital bone, in } \\
\text { the depression between the origins of the sternocleidomastoid and } \\
\text { the trapezius muscles }\end{array}$ & $\begin{array}{l}\text { Perpendicular insertion in the direction of the } \\
\text { opposite eye }\end{array}$ \\
\hline TE16 (Tianyou) & $\begin{array}{l}\text { In the anterior region of the neck, at the same level as the angle of } \\
\text { the mandible, in the depression posterior to the sternocleidomastoid } \\
\text { muscle }\end{array}$ & $\begin{array}{l}\text { Oblique superior insertion across the } \\
\text { sternocleidomastoid muscle }\end{array}$ \\
\hline LI17 (Tianding) & $\begin{array}{l}\text { On the anterior aspect of the neck, at the same level as the cricoid } \\
\text { cartilage, just posterior to the border of the sternocleidomastoid muscle }\end{array}$ & Oblique inferior insertion along the scalene \\
\hline GB21 (Jianjing) & $\begin{array}{l}\text { In the posterior region of the neck, at the midpoint of the line } \\
\text { connecting the spinous process of the } 7 \text { th cervical vertebra (C7) with the } \\
\text { lateral end of the acromion }\end{array}$ & Oblique medial insertion \\
\hline SI14 (Jianwaishu) & $\begin{array}{l}\text { In the upper back region, at the same level as the inferior border of } \\
\text { the spinous process of the 1st thoracic vertebra (T1), } 3 \text { body-cun lateral } \\
\text { to the posterior median line }\end{array}$ & $\begin{array}{l}\text { Oblique inferior insertion in the direction of the } \\
\text { superior part of the medial border of the scapula }\end{array}$ \\
\hline Paravertebral point & $\begin{array}{l}0.5 \text { body-cun lateral to the spinous process at the levels of the } 5 \text { th } \\
\text { and the } 7 \text { th cervical vertebrae, respectively }\end{array}$ & Perpendicular insertion \\
\hline
\end{tabular}




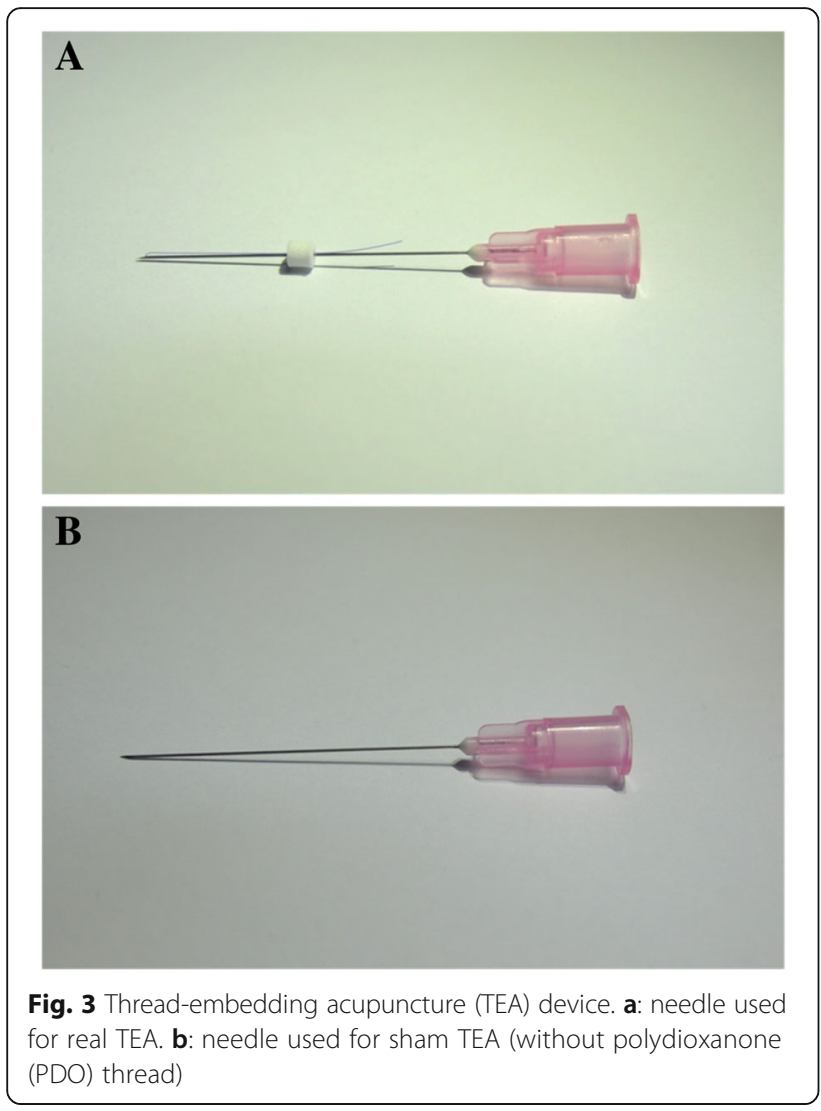

completion of TEA treatment. The 100-mm VAS will be used to assess CNP because it is a fast and straightforward method for evaluating the subjective degree of pain intensity [40]. Participants will be instructed to report the intensity of neck pain experienced within the past week on a linear scale of 0 to 100 (0, no pain; 100, pain as bad as it could be) [41].

\section{Secondary outcomes}

The secondary outcome will consist of pain, clinical relevance, disability, pressure pain threshold, psychological stress, quality of life, global assessment, safety, and blinding test.

\section{Pain}

The secondary outcome for pain will be the mean change on the 100-mm VAS for CNP from the baseline to week 3 , week 6 , and week 8 , respectively.

\section{Clinical relevance}

Clinical relevance will be assessed using the Minimal Clinically Important Difference (MCID), which is the minimal change in the evaluation score that is clinically meaningful for patients [42]. One should note that a statistically significant difference between two groups may not always be clinically significant. Several previous studies have reported the MCID on the VAS for chronic pain as $15 \mathrm{~mm}$ [43-45]. According to a consensus statement of the MCID recommendation, a decrease greater than 30 and $50 \%$ in the VAS score is considered as a moderate clinically important change and a substantial improvement, respectively [46]. Based on these studies, the clinical relevance for CNP will be evaluated by using the ratio of the number of patients with decreases on the VAS $\geq 15 \mathrm{~mm}$ or with percentiles $\geq 30 \%$ and $\geq 50 \%$ relative to baseline to the total number of patients, respectively.

\section{Disability}

The Neck Disability Index (NDI) will be used to measure functional disability in the neck [47]. The validated Korean version of the NDI will be used for this trial [48]. The NDI is the most widely used questionnaire to evaluate cervical pain and dysfunction in daily life and consists of 10 questions, with a 6-point Likert scale from 0 to 5 points. The overall score ranges from 0 to 50 points, and a higher score indicates a greater degree of perceived functional disability.

\section{Pressure pain threshold}

A Digital Pressure Algometer device (Wagner Pain Test ${ }^{\mathrm{Tm}}$ - Model FPX-25 (WAGNER INSTRUMENTS, Greenwich, CT, USA)) will be used to measure the pressure pain threshold (PPT) at three points on both sides: the bilateral levator scapulae, trapezius descendens, and paravertebral point of the sixth cervical spine [49]. The PPT will be measured twice at each point, and the mean value $\left(\mathrm{kg} / \mathrm{cm}^{2}\right)$ will be used.

\section{Psychological stress}

The Hospital Anxiety and Depression Scale (HADS) is a simple self-assessment scale to detect anxiety and depression in patients and has been shown to be reliable and valid [50]. The HADS consists of a total of 14 items: a seven-item depression scale and a seven-item anxiety scale. Both anxiety and depression are known to be correlated with CNP [51-53]. The Korean version of the HADS will be used for this trial [54].

\section{Quality of life}

The Korean version of the EuroQol 5-Dimension (EQ-5D) questionnaire will be used for evaluating the quality of life (QOL) of the participants [55]. The EQ-5D consists of five categories (mobility, personal care, daily activities, pain/ discomfort and anxiety/depression), and each category contains three statements describing personal health status [56]. Participants will instructed to select the most appropriate one among the three statements. 


\section{Global assessment}

The participants' global assessment will be performed using the Patient Global Impression of Change (PGIC), a self-reported 7-point categorical scale that is used to evaluate the overall improvement after treatment [57]. Participants will evaluate themselves as to the improvement of their symptoms at week 6 and week 8 from baseline by selecting one of the following seven options: (1) very much improved, (2) much improved, (3) minimally improved, (4) no change, (5) minimally worse, (6) much worse, or (7) very much worse.

\section{Adverse events and safety}

In this study, various post-intervention responses, such as pain, stiffness, and irritation, will be identified as adverse events related to the TEA treatment. Researchers will record the kinds of these adverse events, as well as the day that the adverse event occurs and is over. The duration of each adverse event will be recorded as a treatment outcome to compare between-group differences. Any causal relationship between the TEA treatment and adverse events will be evaluated using a 6 -grade scale $(1=$ definitely related, $2=$ probably related, $3=$ possibly related, $4=$ probably not related, $5=$ definitely not related, and $6=$ unknown), and the seriousness of the adverse events will be scored using a 4-point Spilker scale $(1=$ mild, $2=$ moderate, $3=$ severe, and $4=$ extremely severe). If any serious adverse event (SAE) occurs, the researcher will immediately inform the SAE to the primary investigator (PI). The allocation status of the subject will be disclosed to the PI only, and the PI will report the SAE to the Ethics Committee so that a decision can be made regarding whether the subject needs to withdraw from the trial. All unexpected and unintended adverse events (AEs) will be recorded at each visit. During the screening and follow-up (week 6) periods, every participants will undergo blood tests, including liver function, blood glucose, and kidney function; every female participant will also receive urine pregnancy tests.

\section{Blinding assessment}

Allocation guessing about the real TEA group vs. the sham TEA group will be assessed after the first treatment and after the last treatment. The subjects will select one of the following three items on a questionnaire about their allocation guess: "classical acupuncture typically used in Korean Medicine clinics," "non-classical acupuncture, rarely used in Korean Medicine clinics," or "don't know" [58].

\section{Statistical methods}

\section{Sample size}

This pilot RCT is designed as a preliminary to a fullscale confirmatory trial. Therefore, we assume that a total of 50 participants will be an acceptable sample size, considering 20 participants in each group with a $20 \%$ drop-out rate.

\section{Statistical analysis}

Data will be analyzed by an independent statistician who is blinded to the group allocation and who will use the Statistical Package for the Social Sciences (SPSS) Version 23.0 (IBM Inc., New York, NY, USA). The analysis set will consist of the Intent-to-treat (ITT) set, Per-protocol (PP) set, and Full Analysis Set (FAS). The ITT set will include all participants as originally allocated after randomization. The PP set will include the participants who completed three or more treatment sessions (75\%) and the entire trial without any major violation of the protocol. Participants who are assessed at least once after random allocation will compose the FAS, which will be used for the main analysis of the treatment outcomes. Safety will be analyzed using the ITT set. The significance level will be set at $p<0.05$. Missing data will be handled using the last-observation-carried-forward (LOCF) method.

The demographic and baseline characteristics, such as age, gender, medical history, duration of CNP and treatment expectancy, will be summarized for each treatment group. Depending on whether a normal distribution is found or not, baseline assessments for homogeneity will be conducted using the Wilcoxon rank sum test or the $t$ test for continuous variables and Fisher's exact test or the chi-square test for categorical variables.

The primary outcome, the mean changes from baseline to week 6 in the VAS scores, will be compared between the two groups by using the $t$ test or analysis of covariance (ANCOVA). If the baseline difference in the VAS score between the two groups is statistically significant, an ANCOVA will be performed with the baseline VAS score as a covariate and the study group as a fixed factor. The paired $t$ test or the Wilcoxon signed rank test will be conducted to compare the treatment outcomes before and after intervention in each group. In addition, a repeated-measures analysis of variance (ANOVA) will be used to further confirm the differences in the trends of the visit. The secondary efficacy outcomes will be evaluated using the Wilcoxon rank sum test or the $t$ test for continuous variables and Fisher's exact test or the chi-square test for categorical variables. For safety assessments, all adverse events will be recorded as frequencies with percentages and durations. The chi-square test or Fisher's exact test will be used to evaluate the statistical significance of between-group differences in the frequencies of adverse events. The duration of each adverse event will be compared between the two groups by using the Wilcoxon rank sum test or the $t$ test. Subgroup analyses based on baseline 
characteristics will also be performed for the primary and the secondary outcomes.

\section{Quality control}

All researchers will undergo special training on recruitment, screening, randomization, assessment, instructions for completing the case report form (CRF), etc., for the purpose of conducting a high-quality clinical trial. Simulations based on the role of each researcher as specified in the pre-defined SOP will also be performed. A qualified clinical research associate (CRA) who is blinded to the subjects' assignment will regularly monitor the overall progress of the on-going research, such as the completion of informed consent forms, the screening process according to the protocol, the recording of adverse events, and the recording and management of data on the CRF. The monitoring process, which is independent from investigator and sponsor, will consist of four phases: a site initiation visit, initiative monitoring visit at first subject enrollment, interim monitoring visits during the clinical trial, and a close-out visit upon completion of clinical trial.

\section{Discussion}

TEA is a new type of acupuncture that can extend the therapeutic stimulation through the use of thread embedded at certain acupoints. Several case reports on the effectiveness of PDO TEA have been published [29, 5961], but, to the best of our knowledge, no RCT investigating the efficacy of the embedded PDO thread in treating patients with CNP has been reported. Therefore, in this trial, we will designate a sham TEA group as a control group to evaluate the differences in the therapeutic effects caused by the PDO thread.

At present, a practitioner-patient-blinded (doubleblinded) sham TEA device has been neither developed nor validated. Therefore, we will use a hollow TEA needle for sham TEA, and will also investigate whether the sham TEA is patient-blinded. In both groups, the hollow needle insertion in the TEA treatment will contribute to relieving neck pain due to the dry-needling effect [62, 63]. Nevertheless, we hypothesize that real PDO TEA will have a superior therapeutic effect because the participant will experience both the continuous stimulation caused by the embedded PDO thread and the initial stimulation caused by the dry-needling.

This study has some limitations. First, the follow-up period of 4 weeks is short. Second, the sample size (50 participants) is small. Third, the practitioner cannot be blinded to the allocation because of the absence of a double-blinded sham device for PDO TEA. Despite these limitations, this study is the first clinical trial to apply both PDO TEA and sham TEA in the treatment of patients with CNP.
The protocol has also been designed in accordance with the Standard Protocol Items: Recommendations for Intervention Trials (SPIRIT) 2013 (see Additional file 2) [64]. Every step of the trial will be rigorously conducted and monitored to ensure methodological integrity and scientific validity. The results of this study will provide valuable data and insights for a confirmative, full-scale $\mathrm{RCT}$ to determine the clinical effects of PDO TEA for the treatment of patients with CNP.

\section{Trial status}

The recruitment of participants for this research began on 22 May 2017, and is presently ongoing. Recruitment is expected to be completed by the end of December 2017.

\section{Additional files}

Additional file 1: Details of polydioxanone thread-embedding acupuncture treatments based on the Standards for Reporting Interventions in Clinical Trials of Acupuncture (STRICTA) Checklist 2010. (DOCX 28 kb)

Additional file 2: Standard Protocol Items; Recommendations for Interventional Trials (SPIRIT) 2013 Checklist: recommended items to address in a clinical trial protocol and related documents*. (DOCX $57 \mathrm{~kb}$ )

\section{Abbreviations}

AE: Adverse event; ANCOVA: Analysis of covariance; ANOVA: Analysis of variance; CNP: Chronic neck pain; CRF: Case report form; DKMHDU: Dunsan Korean Medicine Hospital of Daejeon University; FAS: Full Analysis Set; HADS: Hospital Anxiety and Depression Scale; IRB: Institutional Review Board; ITT: Intent-to-treat; MCID: Minimal Clinically Important Difference; NDI: Neck Disability Index; NSAID: Non-steroidal anti-inflammatory drug; PDO: Polydioxanone; PGIC: Patient Global Impression of Change; PI: Primary investigator; PP: Per protocol; PPT: Pressure pain threshold; RCT: Randomized clinical trial; SAE: Serious adverse event; SOP: Standard operating procedures; TEA: Thread-embedding acupuncture; VAS: Visual analog scale

\section{Acknowledgements}

Not applicable.

\section{Funding}

This study was supported by grants from the project (K18121) of the Korea Institute of Oriental Medicine (KIOM), Republic of Korea. The study execution, data management, statistical analysis, and publication of the results will be performed independently from this funding source.

\section{Availability of data and materials}

Not applicable.

\section{Authors' contributions}

EK and HK, as co-first authors, equally contributed to the study design and the drafting of this manuscript. $\mathrm{CH}$ and YK coordinated this research and participated in the revision of this manuscript as the corresponding authors. $\mathrm{CH}$ and SJ will be project supervisors. EK will conduct the TEA treatment as an individual practitioner. HK will participate in the assessments of the outcomes. All authors have read and approved this final manuscript for submission.

\section{Ethics approval and consent to participate}

This trial has been approved by the Institutional Review Board (IRB) of Korean Medicine Hospital of Daejeon University (DJDSKH-17-BM-08; March 2017, protocol version 1.2). This study protocol has been designed according to Korean Good Clinical Practices (GCPs) and the Declaration of Helsinki, and has been registered with the Clinical Research Information Service (CRIS) of the National Research Institution of Health in Korea (identifier: KCT0002452), which is recognized as a 
registry in the World Health Organization's network. Other important protocol modifications after protocol publication and publication of trial results will be notified through updates on the trial registration site (CRIS). All documents will be identified by an identification code rather than personal information and will be securely stored in a cabinet with locks in the Clinical Trial Center of DKMHDU. Data processing for statistical analyses will be performed by an independent third party and no interim analyses will be conducted.

\section{Consent for publication}

Not applicable.

\section{Competing interests}

The authors declare that they have no competing interests.

\section{Publisher's Note}

Springer Nature remains neutral with regard to jurisdictional claims in published maps and institutional affiliations.

\section{Author details}

${ }^{1}$ Department of Acupuncture and Moxibustion Medicine, College of Korean Medicine, Daejeon University, 62, Daehak-ro, Dong-gu, Daejeon 34520, Republic of Korea. ${ }^{2}$ Department of Acupuncture and Moxibustion Medicine, Dunsan Korean Medicine Hospital of Daejeon University, 75, Daedeok-daero 176 beon-gil, Seo-gu, Daejeon 35235, Republic of Korea. ${ }^{3}$ Clinical Research Division, Korea Institute of Oriental Medicine, 1672, Yuseongdae-ro, Yuseong-gu, Daejeon 34054, Republic of Korea.

\section{Received: 30 October 2017 Accepted: 16 November 2018} Published online: 06 December 2018

\section{References}

1. Hogg-Johnson S, van der Velde G, Carroll LJ, Holm LW, Cassidy JD, Guzman $J$, et al. The burden and determinants of neck pain in the general population: results of the Bone and Joint Decade 2000-2010 Task Force on Neck Pain and Its Associated Disorders. Spine (Phila Pa 1976). 2008;33(4 Suppl):S39-51.

2. Côté $P$, Cassidy JD, Carroll LJ, Kristman V. The annual incidence and course of neck pain in the general population: a population-based cohort study. Pain. 2004;112(3):267-73.

3. Breivik H, Collett B, Ventafridda V, Cohen R, Gallacher D. Survey of chronic pain in Europe: prevalence, impact on daily life, and treatment. Eur J Pain. 2006;10(4):287.

4. Webb R, Brammah T, Lunt M, Urwin M, Allison T, Symmons D. Prevalence and predictors of intense, chronic, and disabling neck and back pain in the UK general population. Spine. 2003:28(11):1195-202.

5. Von Korff M, Crane P, Lane M, Miglioretti DL, Simon G, Saunders K, et al. Chronic spinal pain and physical-mental comorbidity in the United States: results from the national comorbidity survey replication. Pain. 2005;113(3): $331-9$.

6. Son KM, Cho NH, Lim SH, Kim HA. Prevalence and risk factor of neck pain in elderly Korean community residents. J Korean Med Sci. 2013:28(5):680-6.

7. Cote P, van der Velde G, Cassidy JD, Carroll L, Hogg-Johnson S, Holm LW, et al. The burden and determinants of neck pain in workers: results of the Bone and Joint Decade 2000-2010 Task Force on Neck Pain and Its Associated Disorders. Spine (Phila Pa 1976). 2008;33(4 Suppl):S60-74

8. Haldeman S, Carroll L, Cassidy JD. Findings from the Bone and Joint Decade 2000 to 2010 Task Force on Neck Pain and its Associated Disorders. J Occup Environ Med. 2010:52(4):424-7.

9. Hoy D, March L, Woolf A, Blyth F, Brooks P, Smith E, et al. The global burden of neck pain: estimates from the global burden of disease 2010 study. Ann Rheum Dis. 2014;73(7):1309-15.

10. Martin BI, Turner JA, Mirza SK, Lee MJ, Comstock BA, Deyo RA. Trends in health care expenditures, utilization, and health status among US adults with spine problems, 1997-2006. Spine. 2009;34(19):2077-84.

11. Enthoven P, Skargren E, Oberg B. Clinical course in patients seeking primary care for back or neck pain: a prospective 5-year follow-up of outcome and health care consumption with subgroup analysis. Spine (Phila Pa 1976). 2004;29(21):2458-65.

12. Borenstein DG. Chronic neck pain: how to approach treatment. Curr Pain Headache Rep. 2007;11(6):436-9.
13. Furlan AD, Yazdi F, Tsertsvadze A, Gross A, Van Tulder M, Santaguida L, et al. Complementary and alternative therapies for back pain II. Evid Rep Technol Assess (Full Rep). Evid Rep Technol Assess (Full Rep). 2010;(194):1-764.

14. Sanders SH, Harden RN, Vicente PJ. Evidence-based clinical practice guidelines for interdisciplinary rehabilitation of chronic nonmalignant pain syndrome patients. Pain Pract. 2005;5(4):303-15.

15. Borghouts J, Janssen H, Koes B, Muris J, Metsemakers J, Bouter L. The management of chronic neck pain in general practice: a retrospective study. Scand J Prim Health Care. 1999:17(4):215-20.

16. committee Tcsrse. The cervical spine. 5th ed. Pennsylvania: Lippincott Williams \& Willkins; 2012

17. Plastaras CT, Schran S, Kim N, Sorosky S, Darr D, Chen MS, et al. Complementary and alternative treatment for neck pain: chiropractic, acupuncture, TENS, massage, yoga, Tai Chi, and Feldenkrais. Phys Med Rehabil Clin N Am. 2011:22(3):521-37 ix.

18. Grazio S, Balen D. Complementary and alternative treatment of musculoskeletal pain. Acta Clinica Croatica. 2011;50(4):513-30.

19. Eisenberg DM, Davis RB, Ettner SL, Appel S, Wilkey S, Van Rompay M, et al. Trends in alternative medicine use in the United States, 1990-1997: results of a follow-up national survey. JAMA. 1998;280(18):1569-75.

20. Committee KAaMSTC. Acupuncture medicine. Seoul: Hanmi Medical Publishing Company; 2016. p. 181-4.

21. Moy RL, Waldman B, Hein DW. A review of sutures and suturing techniques. J Dermatol Surg Oncol. 1992;18(9):785-95.

22. Qin W, Zhao K, Yang H. Effect of acupoint catgut embedding therapy combined with Chinese medicine for nourishing the kidneys and promoting blood circulation and improving blood glucose and lipid levels as well as the pregnancy rate in obese PCOS patients with infertility. Exp Ther Med. 2016;12(5):2909-14.

23. Garcia-Vivas JM, Galaviz-Hernandez C, Fernandez-Retana J, Pedroza-Torres A Perez-Plasencia C, Lopez-Camarillo C, et al. Transcriptomic profiling of adipose tissue in obese women in response to acupuncture catgut embedding therapy with moxibustion. J Altern Complement Med. 2016; 22(8):658-68.

24. Chen GZ, Xu YX, Zhang JW, Liu SH, Guo ZY. Effect of acupoint catgutembedding on the quality of life, reproductive endocrine and bone metabolism of postmenopausal women. Chin J Integr Med. 2010;16(6):498-503.

25. Li X, Liu Y, Zhang $\mathrm{Q}$, Xiang $\mathrm{N}$, He M, Zhong J, et al. Effect of catgut implantation at acupoints for the treatment of allergic rhinitis: a randomized, sham-controlled trial. BMC Complement Altern Med. 2016; 16(1):454

26. Jang $\mathrm{H}$, Woo $\mathrm{C}$, Ahn $\mathrm{H}$, Kwon $\mathrm{H}$. A clinical four case studies on chronic low back pain treated by needle embedding therapy. J Korea CHUNA Manual Med Spine Nerves. 2014;9(2):45-55.

27. Lee SM, Ji YS, Jeon JH, Kim JH, Kim Yl. Effect of needle-embedding and acupuncture therapy on shoulder pain in Behcet disease patient: a case report. J Acupunct Res. 2013:30(4):219-24.

28. Lee JH, Yang TJ, Lee DG, Lee OJ, Wei TS. The effect of needle-embedding therapy on osteoarthritis of the knee combined with korean medical treatment: report of five cases. J Acupunct Res. 2014;31(4):195-204.

29. Kwon K. The analysis on the present condition of thread-embedding therapy papers published in Journal of Korean Medicine. J Korean Med Ophthalmol Otolaryngol Dermatol. 2014;27(4):16-44.

30. Mao JJ, Armstrong K, Farrar JT, Bowman MA. Acupuncture Expectancy Scale: development and preliminary validation in China. Explore J Sci Heal. 2007; 3(4):372-7.

31. Kim YJ, Lee IS, Kim HS, Lee $H$, Park HJ, Lee $H$, et al. Validation of the Korean version of the Acupuncture Expectancy Scale. Acupunct Med. 2014;32(1):51-5

32. Shen J, Wenger N, Glaspy J, Hays RD, Albert PS, Choi C, et al. Electroacupuncture for control of myeloablative chemotherapy-induced emesis: a randomized controlled trial. JAMA. 2000;284(21):2755-61.

33. Lee S, Nam D, Leem J, Han G, Lee S, Lee J. Efficacy and safety of Myofascialmeridian Release Acupuncture (MMRA) for chronic neck pain: a study protocol for randomized, patient- and assessor-blinded, sham controlled trial. BMC Complement Altern Med. 2016;16:45.

34. Treede R-D, Rief W, Barke A, Aziz Q, Bennett MI, Benoliel R, et al. A classification of chronic pain for ICD-11. Pain. 2015;156(6):1003.

35. Kuorinka I, Jonsson B, Kilbom A, Vinterberg H, Biering-Sørensen F, Andersson $G$, et al. Standardised Nordic questionnaires for the analysis of musculoskeletal symptoms. Appl Ergon. 1987;18(3):233-7. 
36. MacPherson H, Altman DG, Hammerschlag R, Youping L, Taixiang W, White A, et al. Revised STandards for Reporting Interventions in Clinical Trials of Acupuncture (STRICTA): Extending the CONSORT statement. J Evid Based Med. 2010;3(3):140-55

37. Blossfeldt P. Acupuncture for chronic neck pain-a cohort study in an NHS pain clinic. Acupunct Med. 2004:22(3):146-51.

38. Tobbackx Y, Meeus M, Wauters L, De Vilder P, Roose J, Verhaeghe T, et al. Does acupuncture activate endogenous analgesia in chronic whiplashassociated disorders? A randomized crossover trial. Eur J Pain. 2013;17(2): 279-89.

39. Brazil J, Mitchell B, Tu A, Skelton W. Clean needle technique manual for acupuncturists: guidelines and standards for the clean and safe clinical practice. Washington: National Acupuncture Foundation; 1997.

40. Carlsson AM. Assessment of chronic pain. I. Aspects of the reliability and validity of the visual analogue scale. Pain. 1983;16(1):87-101.

41. Hawker GA, Mian S, Kendzerska T, French M. Measures of adult pain: Visual Analog Scale for Pain (VAS Pain), Numeric Rating Scale for Pain (NRS Pain), McGill Pain Questionnaire (MPQ), Short-Form McGill Pain Questionnaire (SFMPQ), Chronic Pain Grade Scale (CPGS), Short Form-36 Bodily Pain Scale (SF-36 BPS), and Measure of Intermittent and Constant Osteoarthritis Pain (ICOAP). Arthritis Care Res (Hoboken). 2011:63(Suppl 11):S240-52.

42. Jaeschke R, Singer J, Guyatt GH. Measurement of health status. Ascertaining the minimal clinically important difference. Control Clin Trials. 1989;10(4): 407-15

43. Kovacs FM, Abraira V, Royuela A, Corcoll J, Alegre L, Tomas M, et al. Minimum detectable and minimal clinically important changes for pain in patients with nonspecific neck pain. BMC Musculoskelet Disord. 2008;9:43

44. Hagg O, Fritzell P, Nordwall A, Swedish Lumbar Spine Study Group. The clinical importance of changes in outcome scores after treatment for chronic low back pain. Eur Spine J. 2003;12(1):12-20.

45. Wolfe F, Michaud K. Assessment of pain in rheumatoid arthritis: minimal clinically significant difference, predictors, and the effect of anti-tumor necrosis factor therapy. J Rheumatol. 2007;34(8):1674-83.

46. Dworkin RH, Turk DC, Wyrwich KW, Beaton D, Cleeland CS, Farrar JT, et al. Interpreting the clinical importance of treatment outcomes in chronic pain clinical trials: IMMPACT recommendations. J Pain. 2008;9(2):105-21.

47. Vernon H, Mior S. The Neck Disability Index: a study of reliability and validity. J Manip Physiol Ther. 1991;14(7):409-15.

48. Song KJ, Choi BW, Choi BR, Seo GB. Cross-cultural adaptation and validation of the Korean version of the Neck Disability Index. Spine (Phila Pa 1976). 2010;35(20):E1045-9.

49. Irnich D, Behrens N, Molzen H, Konig A, Gleditsch J, Krauss M, et al. Randomised trial of acupuncture compared with conventional massage and "sham" laser acupuncture for treatment of chronic neck pain. BMJ. 2001; 322(7302):1574-8.

50. Zigmond AS, Snaith RP. The Hospital Anxiety and Depression Scale. Acta Psychiatr Scand. 1983;67(6):361-70.

51. Blozik E, Laptinskaya D, Herrmann-Lingen C, Schaefer H, Kochen MM, Himmel W, et al. Depression and anxiety as major determinants of neck pain: a cross-sectional study in general practice. BMC Musculoskelet Disord. 2009;10:13.

52. Carroll L, Hogg-Johnson S, van der Velde G, Haldeman S, Holm LW, Carragee EJ, et al. Course and prognostic factors for neck pain in the general population: results of the Bone and Joint Decade 2000-2010 Task Force on Neck Pain and Its Associated Disorders. Spine (Phila Pa 1976). 2008;33(4 Suppl):S75-82.

53. Croft PR, Lewis M, Papageorgiou AC, Thomas E, Jayson MI, Macfarlane GJ, et al. Risk factors for neck pain: a longitudinal study in the general population. Pain. 2001;93(3):317-25.

54. Oh SM, Min KJ, Park DB. A study on the standardization of the Hospital Anxiety and Depression Scale for Koreans: a comparison of normal, depressed and anxious groups. J Korean Neuropsychiatr Assoc. 1999;38(2): 289-96

55. Kim M-H, Cho Y-S, Uhm W-S, Kim S, Bae S-C. Cross-cultural adaptation and validation of the Korean version of the EQ-5D in patients with rheumatic diseases. Qual Life Res. 2005;14(5):1401-6.

56. Rabin R, de Charro F. EQ-SD: a measure of health status from the EuroQol Group. Ann Med. 2001;33(5):337-43.

57. Farrar JT, Young JP, LaMoreaux L, Werth JL, Poole RM. Clinical importance of changes in chronic pain intensity measured on an 11-point numerical pain rating scale. Pain. 2001;94(2):149-58.
58. Bang $H$, Ni L, Davis CE. Assessment of blinding in clinical trials. Control Clin Trials. 2004;25(2):143-56

59. Yun Y-H, Cho S-P, Choi I-H. Case report: correction of nasolabial fold with needle embedding therapy. J Korean Med Ophthalmol Otolaryngol Dermatol. 2011;24(3):154-61.

60. G-s K, Park J-A, Noh J-H, C-h K. Case study of oriental medicine treatment with Mae-sun therapy of the spinal cord injury due to lumbar burst fracture. J Pharmacopuncture. 2010;13(1):129-44.

61. Bae D-B, Park J-H, Lyu Y-S, Lee G-E, Jung H-G, Kang H-W, et al. The case report of 3 dementia patients treated by needle-embedding therapy. J Orient Neuropsychiatry. 2012;23(3):99-116.

62. Mejuto-Vázquez MJ, Salom-Moreno J, Ortega-Santiago R, TruyolsDomínguez S, Fernández-de-las-Peñas C. Short-term changes in neck pain widespread pressure pain sensitivity, and cervical range of motion after the application of trigger point dry needling in patients with acute mechanical neck pain: a randomized clinical trial. J Orthop Sports Phys Ther. 2014;44(4): 252-60.

63. Liu L, Huang Q-M, Liu Q-G, Ye G, Bo C-Z, Chen M-J, et al. Effectiveness of dry needling for myofascial trigger points associated with neck and shoulder pain: a systematic review and meta-analysis. Arch Phys Med Rehabil. 2015;96(5):944-55.

64. Chan A-W, Tetzlaff JM, Altman DG, Laupacis A, Gøtzsche PC, Krleža-Jerić K, et al. SPIRIT 2013 statement: defining standard protocol items for clinical trials. Ann Intern Med. 2013;158(3):200-7.
Ready to submit your research? Choose BMC and benefit from:

- fast, convenient online submission

- thorough peer review by experienced researchers in your field

- rapid publication on acceptance

- support for research data, including large and complex data types

- gold Open Access which fosters wider collaboration and increased citations

- maximum visibility for your research: over $100 \mathrm{M}$ website views per year

At BMC, research is always in progress.

Learn more biomedcentral.com/submissions 